\title{
Tidal effects on groundwater dynamics in unconfined aquifers
}

\author{
B. Ataie-Ashtiani, ${ }^{1 *}$ R. E. Volker ${ }^{2}$ and D. A. Lockington ${ }^{2}$ \\ ${ }^{1}$ Department of Civil Engineering, Sharif University of Technology, Tehran, Iran \\ 2 Department of Civil Engineering, University of Queensland, Queensland 4072, Australia
}

\begin{abstract}
:
The variation of seawater level resulting from tidal fluctuations is usually neglected in regional groundwater flow studies. Although the tidal oscillation is damped near the shoreline, there is a quasi-steady-state rise in the mean water-table position, which may have an influence on regional groundwater flow. In this paper the effects of tidal fluctuations on groundwater hydraulics are investigated using a variably saturated numerical model that includes the effects of a realistic mild beach slope, seepage face and the unsaturated zone. In particular the impact of these factors on the velocity field in the aquifer is assessed. Simulations show that the tidal fluctuation has substantial consequences for the local velocity field in the vicinity of the exit face, which affects the nearshore migration of contaminant in coastal aquifers. An overheight in the water table as a result of the tidal fluctuation is observed and this has a significant effect on groundwater discharge to the sea when the landward boundary condition is a constant water level. The effect of beach slope is very significant and simplifying the problem by considering a vertical beach face causes serious errors in predicting the water-table position and the groundwater flux. For media with a high effective capillary fringe, the moisture retained above the water table is important in determining the effects of the tidal fluctuations. Copyright (c) 2001 John Wiley \& Sons, Ltd.
\end{abstract}

KEY WORDS coastal aquifer; groundwater flow; numerical model; seepage face; tidal fluctuation; variably saturated flow

\section{INTRODUCTION}

Groundwater dynamics near the coast are of interest in aquifer management. In particular a proper assessment of groundwater hydraulics in the coastal zone is necessary for controlling the intrusion of saltwater and controlling or mitigating the effects of nutrients and other contaminants on coastal aquifers, and the adjacent marine environment.

Usually, regional groundwater flow and contaminant transport studies in the vicinity of the coastal zone assume that the coastal boundary water level is equivalent to the mean sea level and that tidal- and waveinduced variations have negligible effect. As the position of a beach groundwater table is an important factor in cross-shore sediment transport and beach stability on a sandy beach, a significant amount of work on beach groundwater has been done by coastal researchers who have tended to concentrate on the nearshore water-table position and its transient variations. Grant (1948) noted that a high groundwater table accelerates offshore sediment transport and beach erosion, and conversely, a low groundwater table may result in pronounced aggradation of the foreshore.

A limited number of researchers have attempted to model the physics of groundwater flow processes in beaches. Dominick et al. (1971) used an implicit finite-difference numerical solution of the Boussinesq equation to simulate beach water-table response to tidal forcing. Their model was for a beach with a vertical face and, as shall be demonstrated, this produces substantial differences from the real case of a sloping beach when predicting water tables and discharges.

* Correspondence to: B. Ataie-Ashtiani, Department of Civil Engineering, Sharif University of Technology, Tehran, Iran. 
Fang et al. (1972) used a two-dimensional finite-element model to solve the beach water-table response to tidal fluctuations. They considered a homogeneous beach with a vertical face. Li et al. (1997) presented a boundary element model for simulating tidal induced fluctuations of the beach groundwater table. The model solves the two-dimensional fully saturated flow equation subject to free and moving boundary conditions, including the seepage dynamics at the beach face. Baird and Horn (1996) have reviewed previous works on groundwater behaviour in sandy beaches and discussed how models of beach groundwater process can be improved by using a numerical approach.

All these works were concerned particularly with the relationship between tides and beach water-tables emphasizing the tidal-induced fluctuations of the water table near the shore and the consequences for processes affecting beach stability. None of them give an accurate picture of groundwater hydraulics and seepage velocity patterns near the sea boundary.

Philip (1973) showed for the first time that a sinusoidal tidal motion on a vertical beach would cause an inland water table over-height relative to mean sea level. Before him a number of investigators (e.g. Jacob, 1950) used a linearization of the Boussinesq equation to study the influence of tides on groundwater level in coastal regions and the result of this linear analysis is a constant groundwater level, at points far enough inland, in equilibrium with the mean sea level. Philip showed that for an aquifer with a horizontal impermeable base, a vertical interface between sea and land, and zero net discharge through the system, the linear analysis may be considerably in error and the groundwater levels are significantly above the mean sea level. Smiles and Stokes (1976) confirmed Philip's prediction using a Hele-Shaw experiment that models groundwater flow. The physical explanation for the phenomenon is that with high water levels the effective transmissivity of the aquifer is greater and so water flows in from the sea more readily than it flows out at low tide (Knight, 1981).

Parlange et al. (1984) used second-order theory to describe the propagation of steady periodic motion in a porous medium, driven by the oscillating level of a reservoir in contact with it. Nielsen (1990) found an analytical solution to the one-dimensional Boussinesq equation for the sloping beach case using a perturbation technique. He showed that real beaches that are sloping produce an additional overheight as a result of the slope. He explained that the asymmetry of the tidal infiltration/draining process for a sloping beach results in a further rise of groundwater level. Nielsen (1990) acknowledged that if decoupling of water table and sea level occurs, the analytical solution will probably fail.

Analytical approaches to model coastal water table fluctuations usually are based on the one-dimensional Boussinesq equation or its approximations. In addition to assumptions such as uniform thickness of aquifer, uniform hydraulic conductivity and a single inland boundary condition at which water-table oscillations reduce to zero, all analytical models are based on the assumption that the exit point of the water table on the beach face is coupled with the tidal sea level.

In cases where there is a constant water level at the landward end of the aquifer, the overheight resulting from tidal pumping may have a significant effect on groundwater discharge to the sea and consequently on contaminant transport. This effect will be magnified where the aquifer is shallow and therefore the effects of tidal fluctuations are more significant. This problem has not been addressed previously, because of the limitations of the analytical solutions (Philip, 1973; Nielsen, 1990) and the assumption that the landward boundary is far inland. Also none of the previous numerical simulations (Dominick et al., 1971; Li et al., 1997) have discussed this problem.

In addition, most of the previous research, including the numerical studies, has focused on the behaviour of the water table in coastal beaches. This is because the problem has been studied largely from the coastal researcher point of view (Dominick et al., 1971; Li et al., 1997), whereas the present work concentrates on the hydrological and environmental engineering point of view, including, for example, the effects of tidal fluctuation on the groundwater flow pattern and its implications for groundwater discharge as a consequence of water table overheight and other factors. The only work in this regard has been done by Turner et al. (1996), who show that neglecting the effects of tidal fluctuations will lead to errors in predictions of groundwater 
discharge to the ocean. However, in their numerical simulation the complexities of the beach face such as mild beach slope, seepage face and the unsaturated zone were neglected.

The major objective of this present investigation is to develop an improved understanding of groundwater flow patterns in unconfined coastal aquifers, with emphasis on the consequences of tidal fluctuations, including the effects of mild beach slope, presence of a seepage face and the unsaturated zone. A proper assessment of groundwater hydraulics in the coastal zone is necessary for controlling or mitigating the impacts of nutrients and other contaminants on coastal wetlands, coastal aquifers and the adjacent marine environment.

The emphasis of the present investigation is on effects of tidal fluctuation on shallow aquifers, which commonly are an important source of freshwater in coastal areas and islands. The results and conclusions may be applicable, to some extent, for deeper aquifers, however, which are affected by large-amplitude tides.

The effects of wave run-up and set-up are not considered in this study. Neglecting their effects is applicable at a macrotidal beach. For a microtidal beach where wave effects are important, however, the present numerical model can include them, provided that the elevation of the sea surface moving with tides and waves is well described.

In this study, the higher density of sea water is not included. Although the higher density of sea water would result in a complex distribution of salt at the seaward end of the aquifer, it is not expected to have a major influence on the flow pattern. This is because the gradients generated by rising and falling tides will be substantially larger than gradients and consequential velocity components owing to variable density effects. The modified numerical model is also used to investigate the effects of tidal fluctuations on seawater intrusion in unconfined aquifers by Ataie-Ashtiani et al. (1999a).

\section{MATHEMATICAL MODEL}

The variably saturated flow problem is solved using a modified version of the SUTRA model (Voss, 1984). The details of the modifications and validation are presented in Ataie-Ashtiani et al. (1999b). The model, which is based on a hybrid finite-element and finite-difference (HFEFD) scheme, has the following advantages:

1. Celia et al. (1990) observed that lumping the mass storage matrix prevents oscillations in the finite-element solution of the variably saturated flow problem. Spatial approximation of the variably saturated flow equation using the HFEFD method is naturally mass lumped, in contrast to common formulations of the finite-element method, which require mass lumping to avoid oscillatory solutions.

2. The HFEFD method has all the advantages of finite-element methods in accommodating irregular geometries and complex boundary conditions.

The governing equation describing the SUTRA fluid mass balance was written in pressure-based form as (Voss, 1984)

$$
\frac{\partial}{\partial x_{i}}\left[\frac{k_{i j} k_{\mathrm{r}} \rho}{\mu}\left(\frac{\partial \rho}{\partial x_{j}}-\rho g e_{j}\right)\right]=\left(S_{\mathrm{w}} \rho S_{\mathrm{op}}+\varepsilon \rho \frac{\mathrm{d} S_{\mathrm{w}}}{\mathrm{d} p}\right) \frac{\partial p}{\partial t}+\varepsilon S_{\mathrm{w}} \frac{\partial p}{\partial c} \frac{\partial c}{\partial t}-Q_{\mathrm{p}}
$$

where $p$ is pressure, $c$ is solute concentration of fluid as a mass fraction, $k_{i j}$ is the permeability tensor, $k_{\mathrm{r}}$ is the relative permeability with respect to the water phase, $x_{i}(i=1,2)$ are Cartesian coordinates, $t$ is time, $e_{j}$ is the unit gravitational vector in the direction of $x_{2}$ (assumed to be vertically upward), $Q_{\mathrm{p}}$ is fluid mass source, $S_{\mathrm{w}}$ is degree of saturation, $S_{\mathrm{op}}$ is the specific pressure storativity, $\rho$ is fluid density, $\mu$ is fluid viscosity, $\varepsilon$ is porosity and $g$ is gravitational acceleration.

In this work SUTRA has been modified to solve the mixed form of the variably saturated flow equation

$$
\frac{\partial}{\partial x_{i}}\left[\frac{k_{i j} k_{\mathrm{r}} \rho}{\mu}\left(\frac{\partial \rho}{\partial x_{j}}-\rho g e_{j}\right)\right]=S_{\mathrm{w}} \rho S_{\mathrm{op}} \frac{\partial p}{\partial t}+\varepsilon \rho \frac{\mathrm{d} S_{\mathrm{w}}}{\mathrm{d} t}+\varepsilon S_{\mathrm{w}} \frac{\partial p}{\partial c} \frac{\partial c}{\partial t}-Q_{\mathrm{p}}
$$


The modified Picard iterative procedure for the mixed form of the variably saturated flow equation is fully mass conserving in the unsaturated zone. By contrast the pressure-based formulation originally in SUTRA exhibits poor mass-balance behaviour (Celia et al., 1990).

The van Genuchten (1980) soil-water equations are used for unsaturated soil hydraulic properties

$$
\begin{aligned}
S_{\mathrm{w}} & =\left[\frac{1}{1+(\alpha|h|)^{n}}\right]^{m} \\
k_{\mathrm{r}}\left(S_{\mathrm{w}}\right) & =S_{\mathrm{w}}^{(1 / 2)}\left[1-\left(1-S_{\mathrm{w}}^{1 / m}\right)^{m}\right]^{2}
\end{aligned}
$$

in which $h$ is pressure head and $\alpha, n$ and $m$ are fitting parameters, with $m$ related to $n$ by $m=1-1 / n$. Degree of saturation is defined as $\left(\theta-\theta_{\mathrm{r}}\right) /\left(\theta_{\mathrm{s}}-\theta_{\mathrm{r}}\right)$, where $\theta$ is water content and $\theta_{\mathrm{r}}$ and $\theta_{\mathrm{s}}$ are residual and saturated water contents.

The general expressions for flow boundary conditions are given by

$$
\begin{aligned}
p\left(x_{i}, t\right) & =p_{\mathrm{BC}} \text { on } B_{1} \\
Q_{\mathrm{p}} & =Q_{\mathrm{BC}} \text { on } B_{2}
\end{aligned}
$$

where $B_{1}$ and $B_{2}$ are the portions of the flow boundary where $p$ and $Q$ are prescribed respectively.

In the present work, the model also has been modified to handle a seepage face boundary condition. A seepage face is an external boundary of the saturated zone where flux is directed outward and pressure is atmospheric. Negative pressures exist above the seepage face. Therefore, all the nodes along the seepage face are treated as Dirichlet nodes, with the prescribed pressure $p=0$. Nodes above the seepage face are specified as no-flow nodes. The position of the seepage face is unknown initially and must be determined using an iterative process and for the non-linear boundary condition. Cooley (1983) modified Neuman's (1973) iterative procedure to determine the seepage-face length. The same procedure is used in this work.

Moreover, to improve the ability of the model to handle the non-linearity of the unsaturated zone equation, an automatic underrelaxation method has been applied for adjustment of pressure after each iteration (Cooley, 1983).

\section{BASIC PROBLEM DESCRIPTION}

In this section the numerical model is applied to study the groundwater behaviour in an aquifer influenced by tidal fluctuations. A schematic representation of the basic problem is presented in Figure 1. The coastal aquifer, $\mathrm{L}=180 \mathrm{~m}$ long and $H=10 \mathrm{~m}$ deep with a $1: 10$ beach slope has no-flow boundaries on the base of the flow domain. For this study the rainfall infiltration has not been included because a short domain length has been considered; thus a no-flow boundary has been applied at the top of the flow domain. The aquifer medium is assumed to be homogeneous and isotropic. Values of the soil parameters used are: horizontal and vertical saturated hydraulic conductivity, $K_{\mathrm{h}}=K_{\mathrm{v}}=8.4 \mathrm{~m} \mathrm{day}^{-1}$; porosity, $\varepsilon=0.3$; residual water content, $\theta_{\mathrm{r}}=0.01$; and specific storage, $S_{\mathrm{s}}=10^{-6} \mathrm{~m}^{-1}$. Also the values of the Van Genuchten soil parameters are: $\alpha=3.3 \mathrm{~m}^{-1}, n=4.1$, which represent a fine sand of reasonably regular grain-size distribution.

The landward water level on the right-hand boundary in Figure 1 is maintained at a constant value of $8.3 \mathrm{~m}$, whereas the seaward boundary is described by a mean seawater level of $7 \mathrm{~m}$, on which is superimposed a sinusoidal semi-diurnal tide with $1 \mathrm{~m}$ amplitude and $12 \mathrm{~h}$ period. Therefore the average regional groundwater gradient is 0.01 .

The water table initially is assumed to vary linearly from the landward boundary water level to mean seawater level at the seaward boundary and a hydrostatic pressure distribution below the water table is assumed, with the pressure head in the unsaturated zone being limited to $-1 \mathrm{~m}$. Based on this initial condition, the steady-state non-tidal condition is calculated and is then applied as the initial condition for the case with a tide.

The finite-element dimensions vary from $0.2 \mathrm{~m}$ in the unsaturated part to $1.5 \mathrm{~m}$ in saturated parts of the aquifer. A smaller element size is used near the seaward and landward boundaries to model, respectively, 


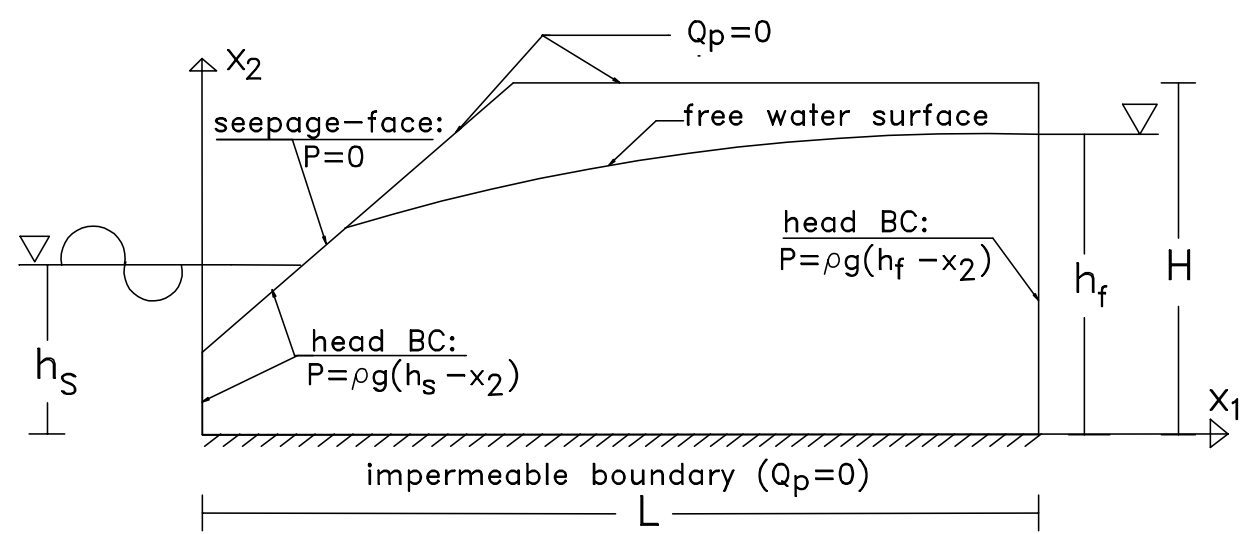

Figure 1. Schematic of the flow domain and boundary conditions (BC) used in the simulations

the seepage face and water level more accurately. The numbers of nodes and elements are 5633 and 5287 respectively. Time step, $\Delta t$, varies from 0.05 to $0.5 \mathrm{~h}$ in the simulations.

To show the effect of different parameters, 13 variations on a basic problem are simulated. Table I shows the parameters in these cases.

\section{RESULTS}

The results of the simulations, after the system has reached the final equilibrium or cyclic steady-state, are presented in this section. Equilibrium is assumed to occur when there is no significant change in pressure head distribution nor in groundwater flux in consecutive periods. Table II gives a comparison of discharges, seepage face heights at low tide and over-height at $x=80 \mathrm{~m}$ for the different cases listed in Table I.

\section{Basic problem (case I)}

Groundwater levels predicted by the numerical model are shown in Figure 2. There is the quasisteady-state pumping up effect of the tide noted by Philip (1973). In addition there is a local transient bulge in the water table landward of the shore. This results from the asymmetry of the tidal infiltration

Table I. Parameter values for the various cases simulated

\begin{tabular}{lcccccc}
\hline \multicolumn{1}{c}{ Case } & $\begin{array}{c}\text { Beach } \\
\text { slope }\end{array}$ & $\begin{array}{c}\text { Tidal } \\
\text { amplitude }(\mathrm{m})\end{array}$ & $\begin{array}{c}\text { Hydraulic } \\
\text { gradient }\end{array}$ & $\begin{array}{c}K_{\mathrm{h}} \\
\left(\mathrm{m} \mathrm{day}^{-1}\right)\end{array}$ & $\begin{array}{c}K_{\mathrm{v}} \\
\left(\mathrm{m} \mathrm{day}^{-1}\right)\end{array}$ & $\begin{array}{c}\alpha \\
\left(\mathrm{m}^{-1}\right)\end{array}$ \\
\hline I-a (basic problem) & $1: 10$ & 0 & $0 \cdot 01$ & $8 \cdot 4$ & $8 \cdot 4$ & $3 \cdot 3$ \\
I-b (basic problem) & $1: 10$ & 1 & $0 \cdot 01$ & $8 \cdot 4$ & $8 \cdot 4$ & $3 \cdot 3$ \\
II-a & $1: 10$ & 0 & $0 \cdot 01$ & 42 & 42 & $3 \cdot 3$ \\
II-b & $1: 10$ & 1 & $0 \cdot 01$ & 42 & 42 & $3 \cdot 3$ \\
III-a & $1: 10$ & 0 & $0 \cdot 01$ & 42 & $8 \cdot 4$ & $3 \cdot 3$ \\
III-b & $1: 10$ & 1 & $0 \cdot 01$ & 42 & $8 \cdot 4$ & $3 \cdot 3$ \\
IV-a & $1: 10$ & 0 & $0 \cdot 01$ & $8 \cdot 4$ & $8 \cdot 4$ & $0 \cdot 66$ \\
IV-b & $1: 10$ & 1 & $0 \cdot 01$ & $8 \cdot 4$ & $8 \cdot 4$ & $0 \cdot 66$ \\
V & $1: 10$ & $2 \cdot 5$ & $0 \cdot 01$ & $8 \cdot 4$ & $8 \cdot 4$ & $3 \cdot 3$ \\
VI-a & $1: 10$ & 0 & $0 \cdot 02$ & $8 \cdot 4$ & $8 \cdot 4$ & $3 \cdot 3$ \\
VI-b & $1: 10$ & $2 \cdot 5$ & $0 \cdot 02$ & $8 \cdot 4$ & $8 \cdot 4$ & $3 \cdot 3$ \\
VII-a & Vertical & 0 & $0 \cdot 01$ & $8 \cdot 4$ & $8 \cdot 4$ & $3 \cdot 3$ \\
VII-b & Vertical & $2 \cdot 5$ & $0 \cdot 01$ & $8 \cdot 4$ & $8 \cdot 4$ & $3 \cdot 3$ \\
\hline
\end{tabular}


Table II. Comparisons of key results for various cases

\begin{tabular}{|c|c|c|c|c|c|}
\hline \multirow[b]{2}{*}{ Case } & \multicolumn{2}{|c|}{ Discharge volume } & \multicolumn{2}{|c|}{ Seepage face at low tide } & \multirow[b]{2}{*}{$\begin{array}{l}\text { Overheight at } \\
x=80 \mathrm{~m}(\mathrm{~m})\end{array}$} \\
\hline & $\begin{array}{l}\text { One tidal } \\
\text { cycle }\left(\mathrm{m}^{3}\right)\end{array}$ & $\begin{array}{c}\text { Constant sea } \\
\text { level }\left(\mathrm{m}^{3}\right)\end{array}$ & $\begin{array}{l}\text { Vertical } \\
\text { (m) }\end{array}$ & $\begin{array}{c}\text { Slope } \\
\text { length }(\mathrm{m})\end{array}$ & \\
\hline I & $0 \cdot 23$ & $0 \cdot 32$ & $0 \cdot 32$ & $3 \cdot 20$ & $0 \cdot 28$ \\
\hline II & $1 \cdot 31$ & 1.62 & 0.2 & $2 \cdot 0$ & $0 \cdot 28$ \\
\hline III & $1 \cdot 31$ & 1.55 & $0 \cdot 32$ & $3 \cdot 2$ & $0 \cdot 23$ \\
\hline IV & $0 \cdot 30$ & $0 \cdot 36$ & $0 \cdot 14$ & 1.4 & $0 \cdot 17$ \\
\hline V & $0 \cdot 11$ & $0 \cdot 32$ & 1.78 & $17 \cdot 8$ & $1 \cdot 36$ \\
\hline VI & $0 \cdot 33$ & $0 \cdot 70$ & 1.88 & $18 \cdot 8$ & $1 \cdot 14$ \\
\hline VII & $0 \cdot 28$ & $0 \cdot 33$ & $0 \cdot 61$ & $0 \cdot 61$ & 0.089 \\
\hline
\end{tabular}

and draining process for a sloping beach. Water can infiltrate vertically into the beach face during the rising tide, but must seep essentially horizontally through the beach during the ebbing tide (Turner et al., 1996).

Figure 3 illustrates the velocity field for the low- and high-tide stages. As seen, there is a major gradient between the overheight water level at low tide and the exit point, and the induced velocity resulting from this gradient is much larger than the velocity field resulting from the regional groundwater gradient. Moreover, from the velocity field in Figure 3 it can be seen that the water which infiltrates into the unsaturated sand during the rising tide is distributed outward from the zone of the local peak in the water table during the falling tide. As far as the authors are aware such velocity patterns have not been presented previously.

The variations in the discharge to or recharge from the sea for cases with and without tidal fluctuation are shown in Figure 4 for this case along with cases II, III and IV, which will be discussed subsequently. A major difference between the results of the cases with and without the tide can be seen. During a tidal period the total groundwater flux is $0.23 \mathrm{~m}^{3}$ compared with $0.32 \mathrm{~m}^{3}$ when the tidal fluctuation is not considered. Obviously the overheight of the groundwater table as a result of tidal activity has changed the hydraulic gradient and consequently the amount of discharge to the sea. Neglecting the effects of tidal fluctuation causes a 39\% error in the amount of water discharged to sea.

The elevations of both the exit point and the tide are plotted in Figure 5. It is clear that there is a seepage face during most of the falling tide period and even in the first stages of the rising tide period. For example, the exit point is about $0.32 \mathrm{~m}$ above the seawater level at low tide. Neglecting this phenomenon causes an underestimation of the water table elevation and hence an overestimation of the amount of outflow to the sea.

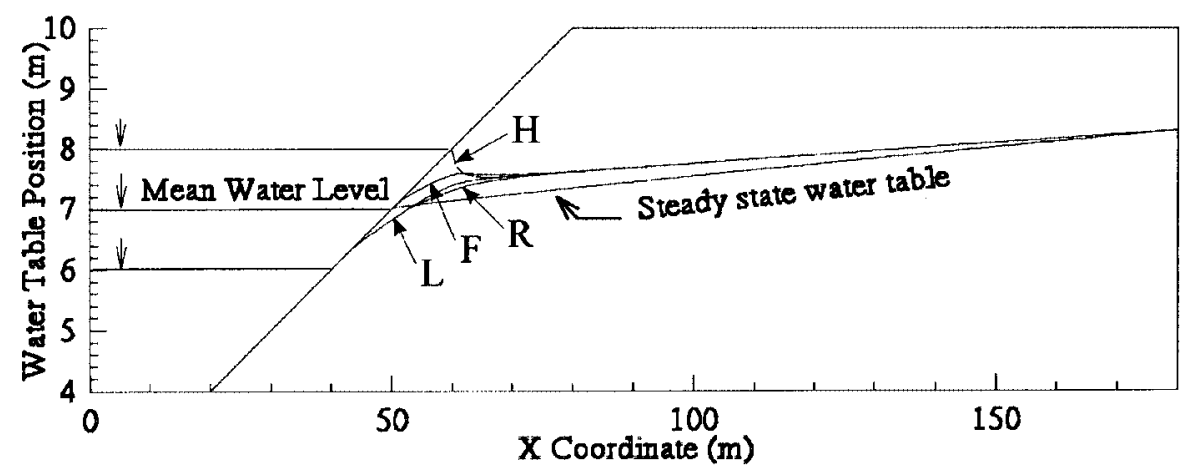

Figure 2. Water tables predicted at different stages of tidal level (case I). H-high tide; F-falling tide; L=low tide; R-rising tide 

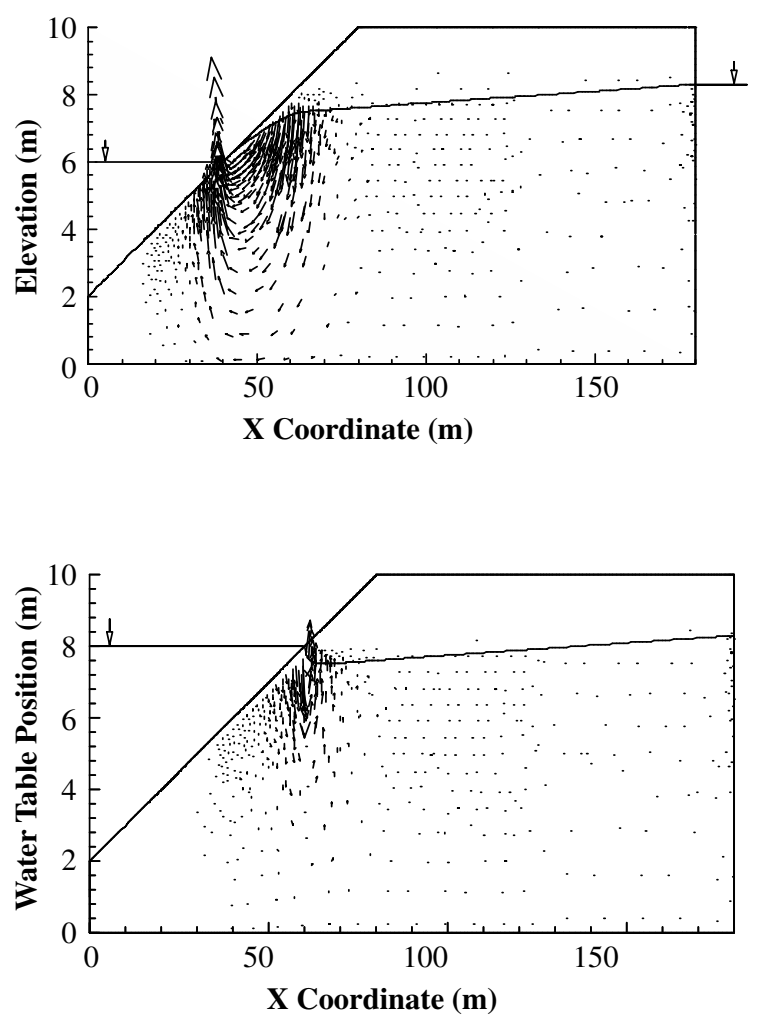

Figure 3. Velocity vectors corresponding to the low and high water level (case I)

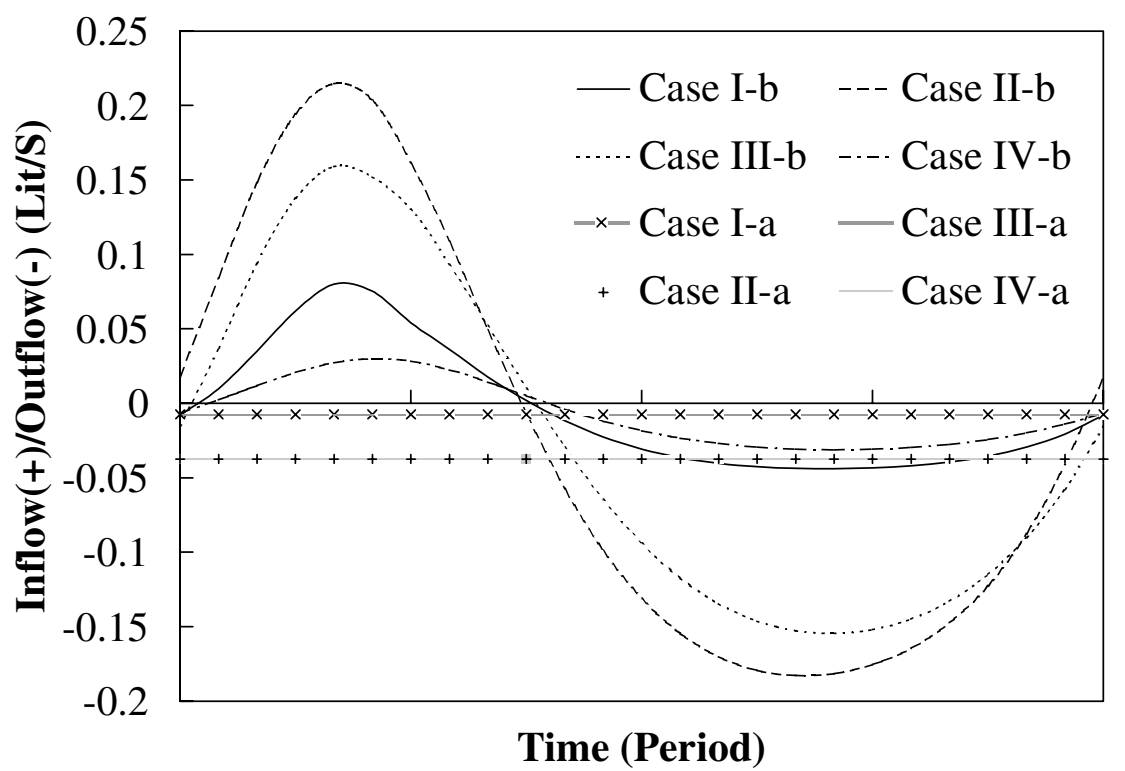

Figure 4. Inflow from $(+)$ and outflow to $(-)$ the sea for the cases with and without tidal fluctuation over 1 tidal cycle 


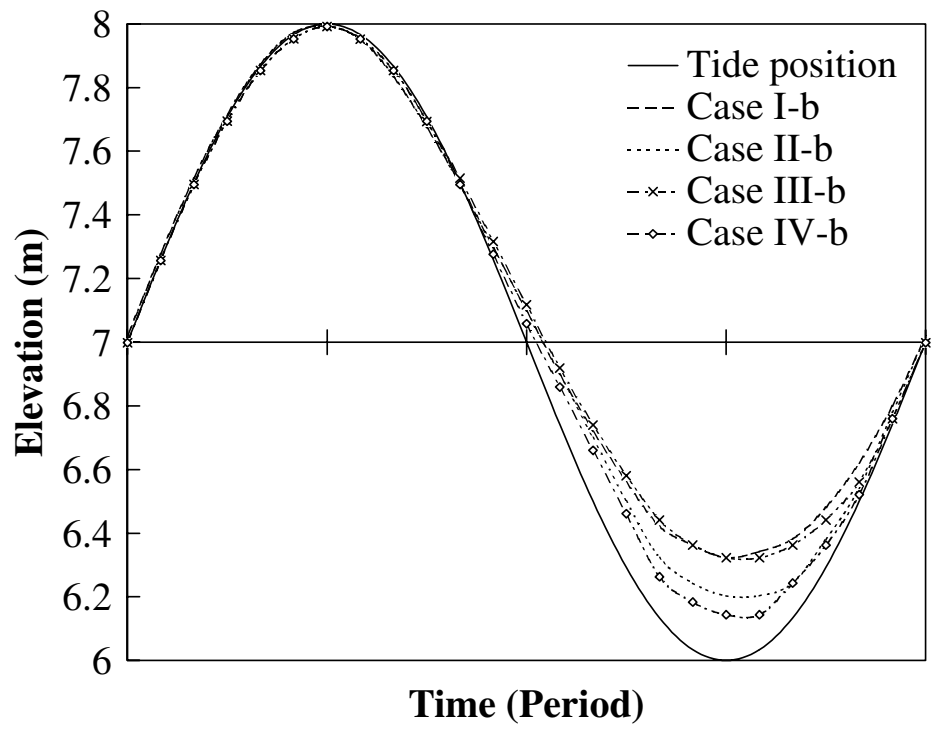

Figure 5. The elevation of the exit point and the tidal water level over a tidal cycle

(a)

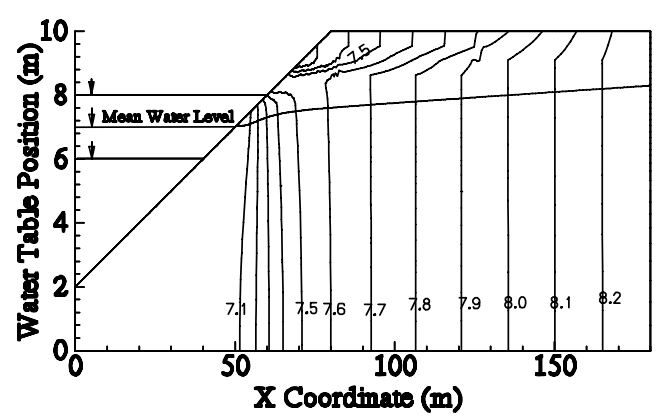

(c)

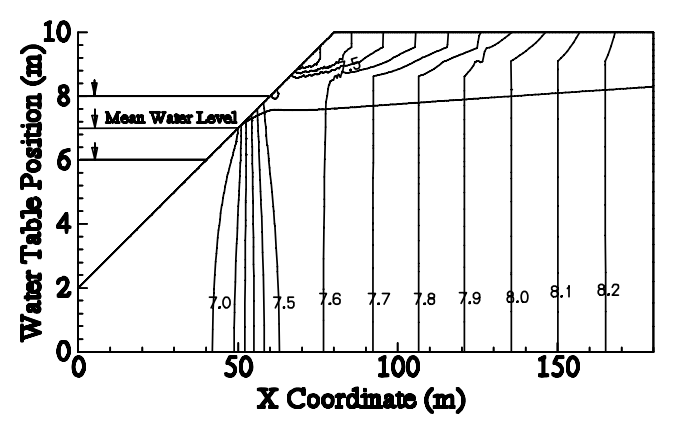

(b)

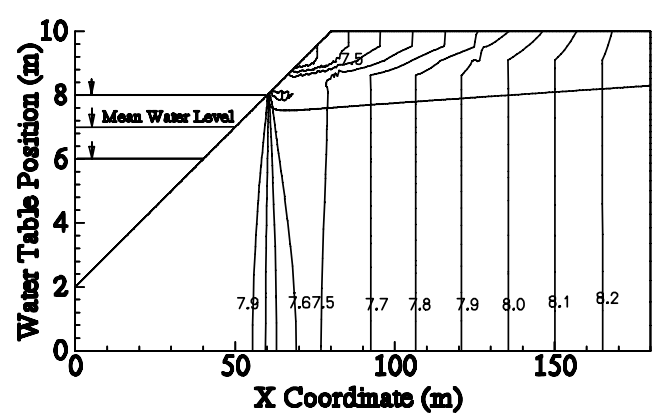

(d)

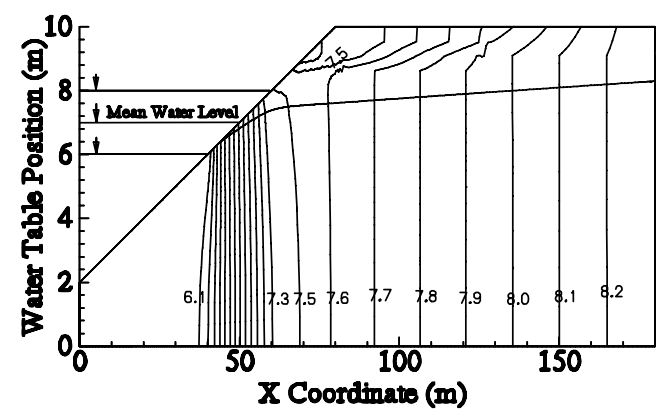

Figure 6. Equipotential lines at (a) rising, (b) high, (c ) falling and (d) low tidal stages for case I

Figure 6 shows the equipotential lines for the groundwater flow at different stages of the tidal fluctuation. The slight irregularities in the 7.6 and $7.9 \mathrm{~m}$ equipotentials are the result of numerical instability in the 
unsaturated zone calculations. Two regimes are evident for the groundwater flow: near the shoreline the equipotential lines are not parallel to each other and they change during a cycle of tidal fluctuation; further inland all equipotential lines are vertical and equally spaced at all stages of tidal fluctuation. The former regime shows the transient effects of tidal fluctuation, whereas the latter one shows effectively steady-state groundwater flow.

\section{Influence of the saturated hydraulic conductivity (cases II and III)}

Figure 7 shows the location of the water table at the various stages of tidal fluctuation for saturated hydraulic conductivity $K_{\mathrm{s}}=42.0 \mathrm{~m} \mathrm{day}^{-1}$. The other parameters are held the same as in the basic problem. Comparison of Figure 2 with Figure 7 shows that as the value of the saturated hydraulic conductivity is increased, the transient effects of tidal fluctuations develop further inland and the steady-state overheight decreases. Figure 5 shows that the seepage face decreases with an increase in saturated hydraulic conductivity because the sand tends to drain at a faster rate for higher saturated hydraulic conductivity and hence the location of the water table is lowered. The exit point is about $0.20 \mathrm{~m}$ above the seawater level at low tide in this case.

The volumes of water discharged to the sea for the cases with and without the tide are $1.31 \mathrm{~m}^{3}$ and $1.62 \mathrm{~m}^{3}$ respectively. There is a $24 \%$ difference between these values. The difference between discharges for the steady-state and tidal cases is less than that for the basic problem. This confirms that the steady-state effect of the tide, measured as an overheight above the water table for the case without tidal fluctuation, is smaller in this case than in case I.

The variation of discharge to the sea during a full tidal cycle is shown in Figure 4 along with the discharge for the case without a tide.

To investigate the effects of anisotropic hydraulic conductivity, case III is considered. This is the same as the basic problem except that the horizontal hydraulic conductivity is $42 \mathrm{~m} \mathrm{day}^{-1}$. The water table positions are shown in Figure 8. The water table shape and the steady-state effects of tidal fluctuation in this case are similar to those for case II, which has the same horizontal hydraulic conductivity as case III, and are in contrast to case I, which has the same vertical hydraulic conductivity as case III. Therefore in cases where an anisotropic medium is approximated by an isotropic one, and where the water table form is the major concern, it is better to use the horizontal hydraulic conductivity as the isotropic one. On the other hand Figure 5 shows that when the position of the exit point is under consideration, then using the vertical hydraulic conductivity as the isotropic one is a better approximation to the problem. In other words, in this latter case, it is the vertical hydraulic conductivity that has a greater influence on the position of the exit point and of the seepage face length.

As expected the variation of flux on the sea boundary (Figure 4) for this case is similar to case II, although some difference is seen and this indicates the significance of vertical movements of groundwater near the shoreline. The total amounts of water discharged to the sea in the cases with and without tide are $1.31 \mathrm{~m}^{3}$ and $1.58 \mathrm{~m}^{3}$ respectively, which are close to the case II values. There is a $21 \%$ error in discharge to sea in this case.

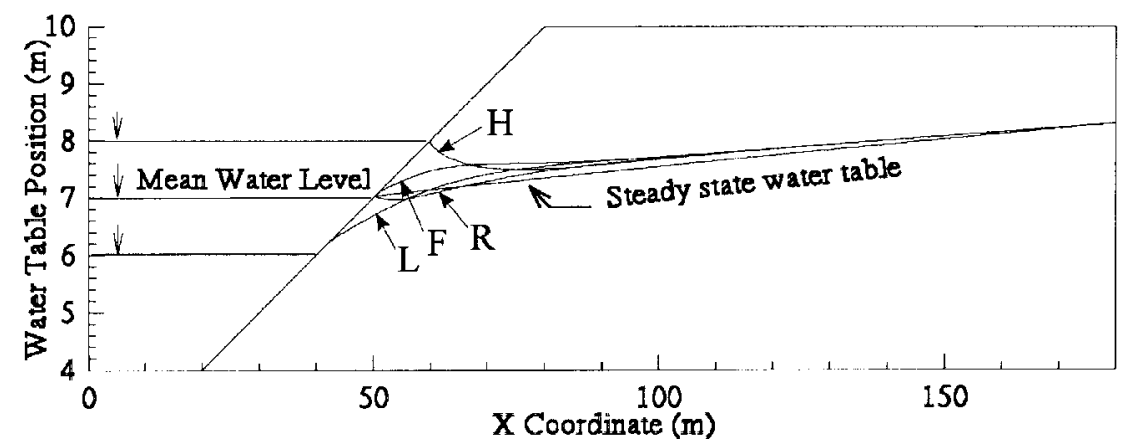

Figure 7. Water tables predicted at different stages of tidal level (case II). H-high tide; F-falling tide; L=low tide; R-rising tide 


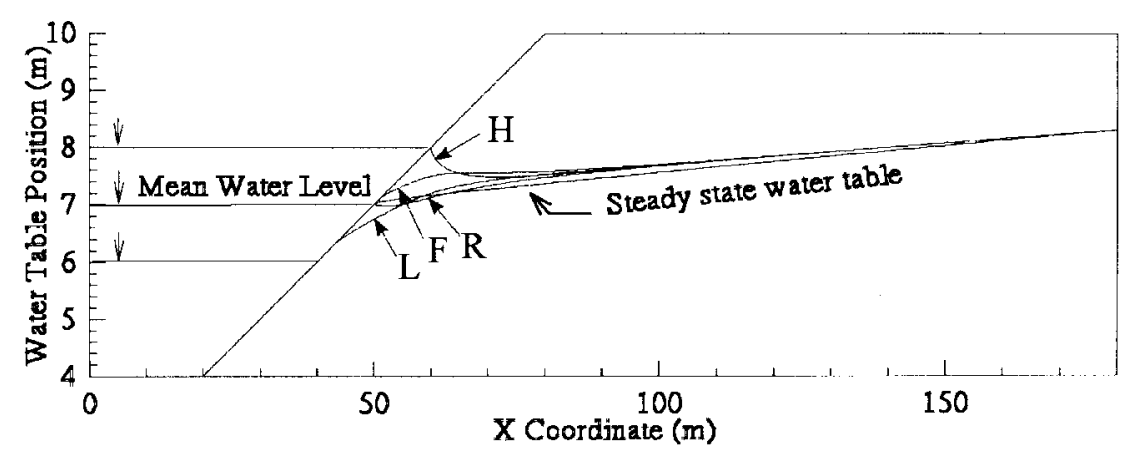

Figure 8. Water tables predicted at different stages of tidal level (case III). H-high tide; F-falling tide; L=low tide; R-rising tide

\section{Influence of unsaturated zone (case IV)}

Figure 9 shows the water-table location at the various stages of tidal fluctuations for a new set of unsaturated zone characteristics. The figure shows the location of the water table for $\alpha=0.66 \mathrm{~m}^{-1}$ and with other parameters as in the basic problem. The effective capillary heights, as defined by El-Kadi and Ling (1993), for the basic and for this case are 0.33 and $1.65 \mathrm{~m}$, respectively. The sensitivity of the water table location (at various stages of tidal fluctuation) to variations of unsaturated characteristics can be observed by comparing Figure 2 with Figure 9. These figures show that, as the value of $\alpha$ increases, the location of the water table is lowered, except at the high-tide stage. As illustrated by Wise et al. (1994), the Van Genuchten parameter $\alpha$ is a measure of absolute pore size. A porous medium with small pores has a small value of $\alpha$ and a large capillary fringe height. Soils with small values of $\alpha$ tend to retain considerable amounts of moisture within the unsaturated zone owing to capillary forces. At small values of $\alpha$, more water is retained under tension, allowing the movement of the surface of the atmospheric pressure well below the region of significant moisture. Moreover, at high tide the water infiltrates more easily for the same reason. Figure 5 also shows that the exit point is lowered, and hence the seepage-face length decreases as the capillary fringe height increases.

Figure 10 is a contour diagram of saturation at low tide predicted by the variably saturated model. The predicted location of the water table at this time is also shown in the figure. This shows that the porous medium retains significant moisture well above the water table in the case where $\alpha=0.66 \mathrm{~m}^{-1}$, whereas the corresponding amount is not significant for the case where $\alpha=3 \cdot 3 \mathrm{~m}^{-1}$.

The volumes of water discharged to the sea for cases with and without tide are $0.30 \mathrm{~m}^{3}$ and $0.36 \mathrm{~m}^{3}$, respectively. The difference between the discharges in these two cases is about $20 \%$. In comparison with the

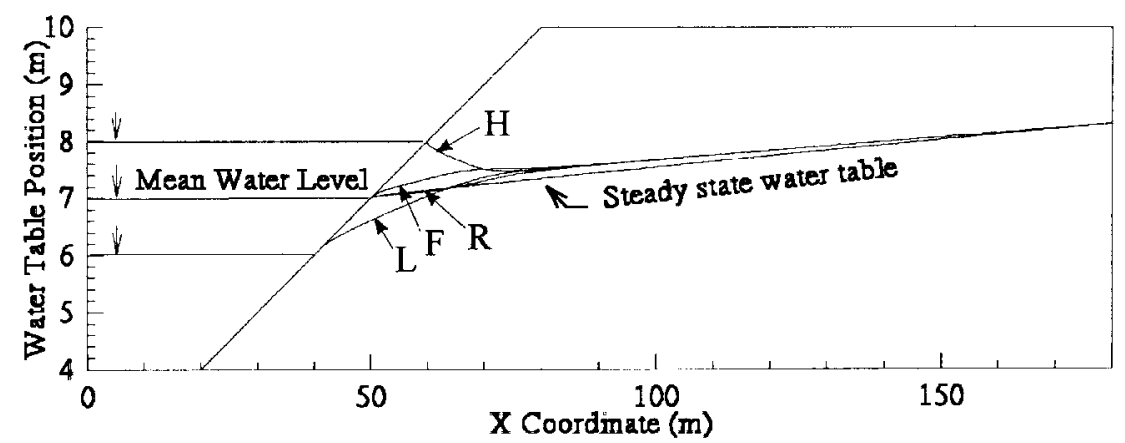

Figure 9. Water tables predicted at different stages of tidal level (case IV). H-high tide; F-falling tide; L=low tide; R-rising tide 
(a)

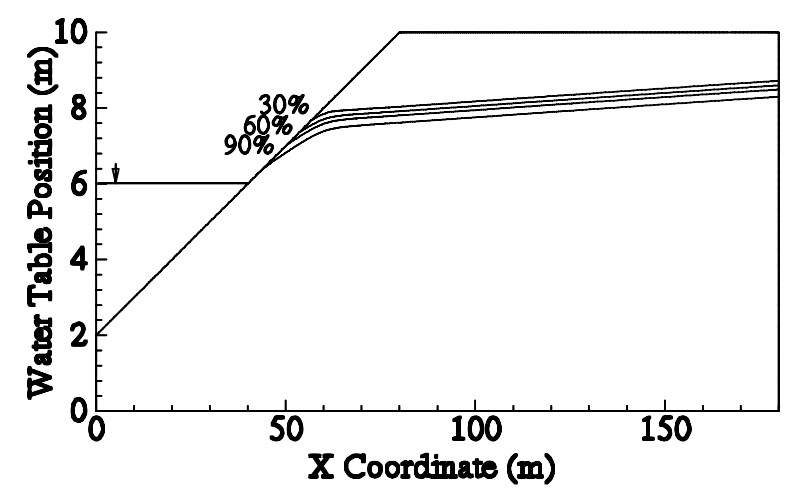

(b)

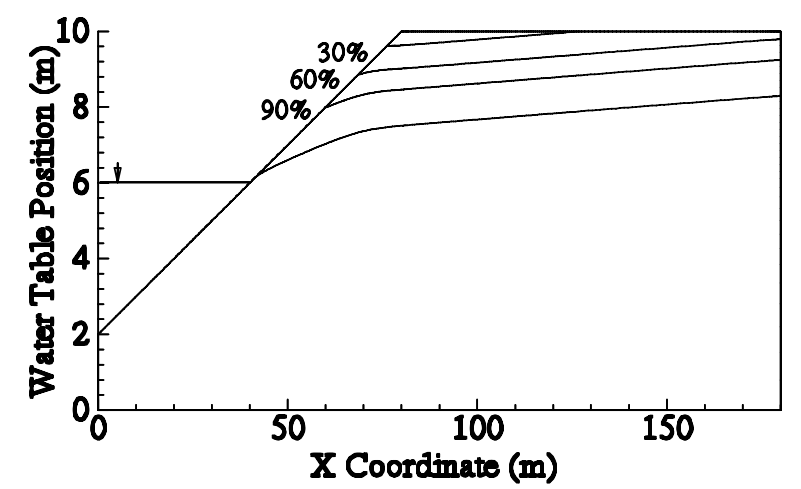

Figure 10. Water tables and contours of effective saturation at 90, 60 and 30\% levels predicted at different low tide stage for (a) case I and (b) case IV

basic problem, this shows that the increase in the effective capillary fringe causes a decrease in the steadystate effects of tidal fluctuation. In other words the water table overheight resulting from tidal fluctuation is decreased. This also can be observed by comparison of Figure 2 and Figure 9.

Figure 4 shows that the variation of discharge to the sea during a period of oscillation is less than that in the basic problem.

\section{Influence of tidal amplitude and regional hydraulic gradient (cases V and VI)}

Obviously the effect of tidal fluctuations on groundwater dynamics is more significant for coastlines where the tidal amplitude is high. The basic problem is changed by increasing the amplitude of tidal fluctuations from $1.0 \mathrm{~m}$ to $2.5 \mathrm{~m}$.

Figure 11 shows the water table positions in this case. As seen in this figure, the strong steady-state effects of tidal fluctuations have reversed the hydraulic gradient from land-sea to sea-land, at least over the length of aquifer used in this case. The situation depicted in Figure 11 is for a constant water level at the inland end; over this distance an initial gradient of 0.01 to mean sea level is insufficient to maintain net discharge 
to the sea in the face of a tidal amplitude of $2.5 \mathrm{~m}$. Obviously if a long enough section of aquifer were included and the steady-state gradient of 0.01 were maintained, eventually a positive gradient to the sea would be established even for a $2.5 \mathrm{~m}$ tide. The overheight eventually would also become negligible at the inland end.

Figure 12 shows the variations in the discharge to and recharge from the sea. The volume of water discharged to the landward source in this case, during a cycle of tidal fluctuation, is $0.11 \mathrm{~m}^{3}$. For similar conditions when the tidal effect is not considered, the hydraulic gradient is from land to sea and the volume of water discharged to the sea is $0.32 \mathrm{~m}^{3}$.

Figure 13 shows that in this case the seepage face is quite significant and cannot be neglected; for example at low tide there is a $1.66 \mathrm{~m}$ difference between seawater level and exit point.

Obviously the regional hydraulic gradient may be increased to overcome the effect of overheight resulting from tidal activity. Figure 14 illustrates the position of the water table for the case where the regional groundwater hydraulic gradient is 0.02 ; in this case the water level at the landward boundary equals $9.6 \mathrm{~m}$. This figure shows that owing to the higher regional gradient, there is still a discharge to the sea in spite of

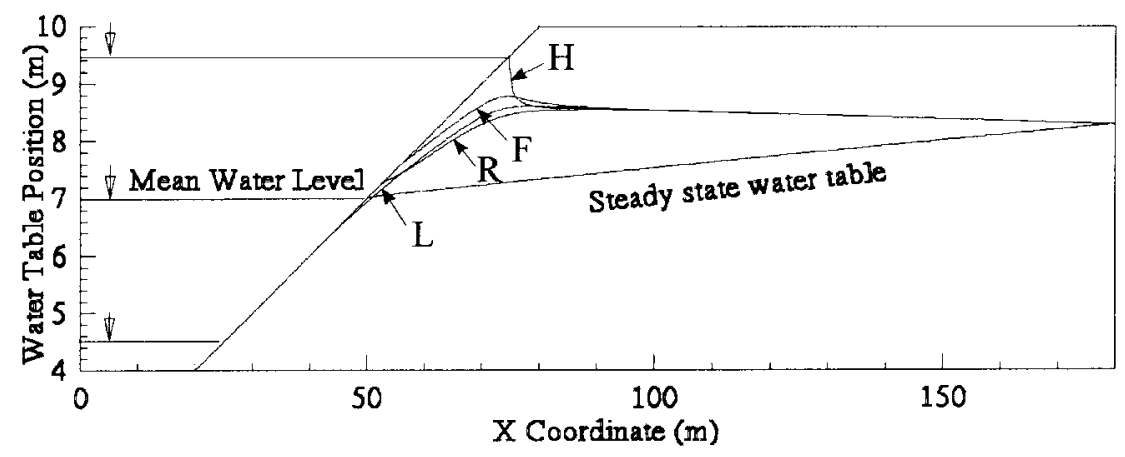

Figure 11. Water tables predicted at different stages of tidal level (case V). H-high tide; F-falling tide; L=low tide; R-rising tide

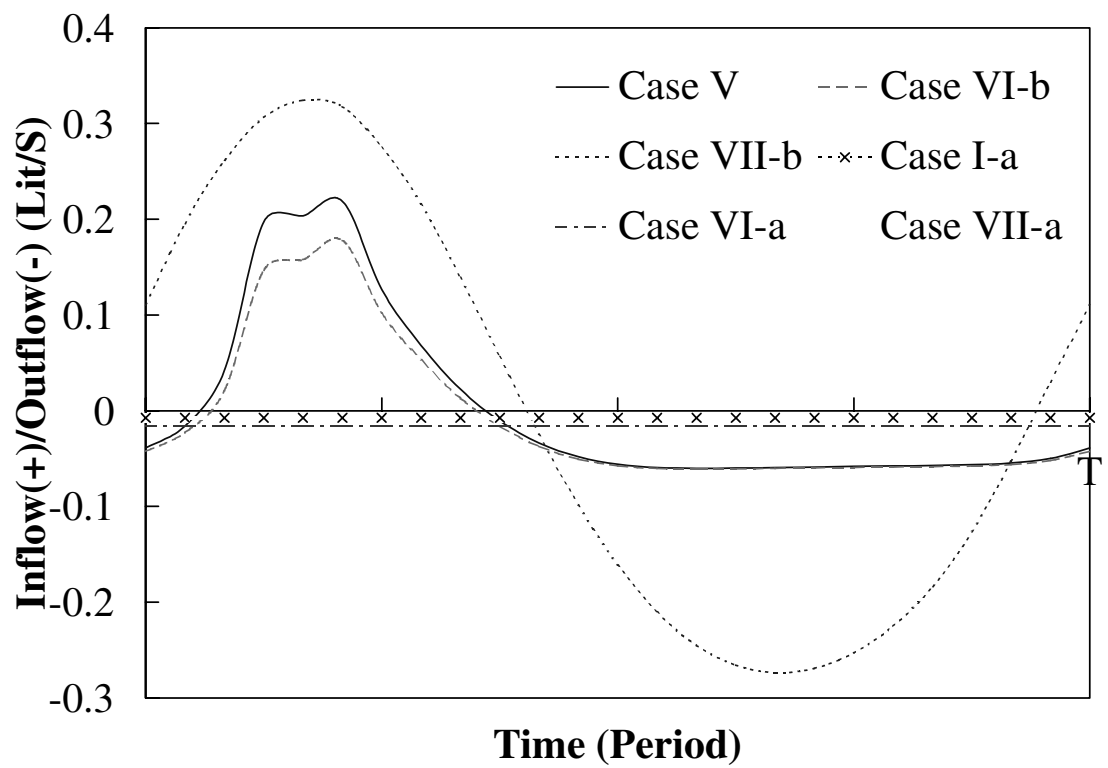

Figure 12. Inflow from $(+)$ and outflow to $(-)$ the sea for the cases with tidal fluctuation and for the case without tidal effect 


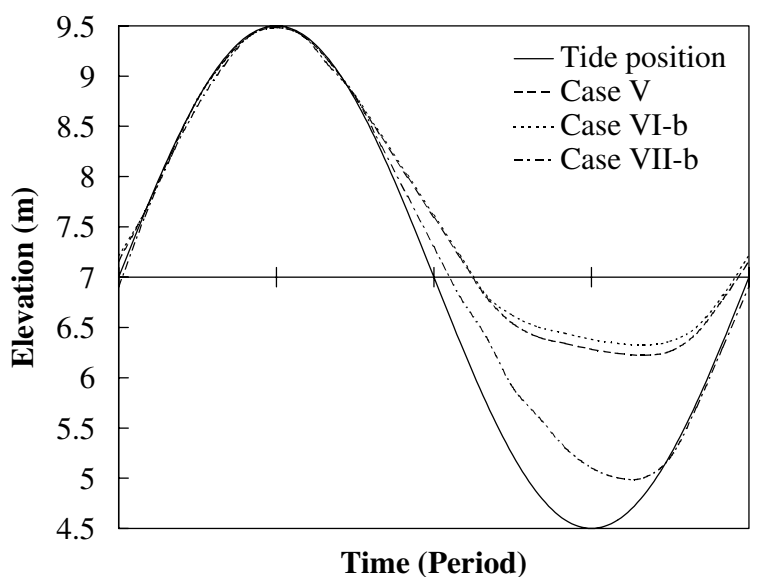

Figure 13. The elevation of the exit point and the tidal water level over 1 tidal cycle

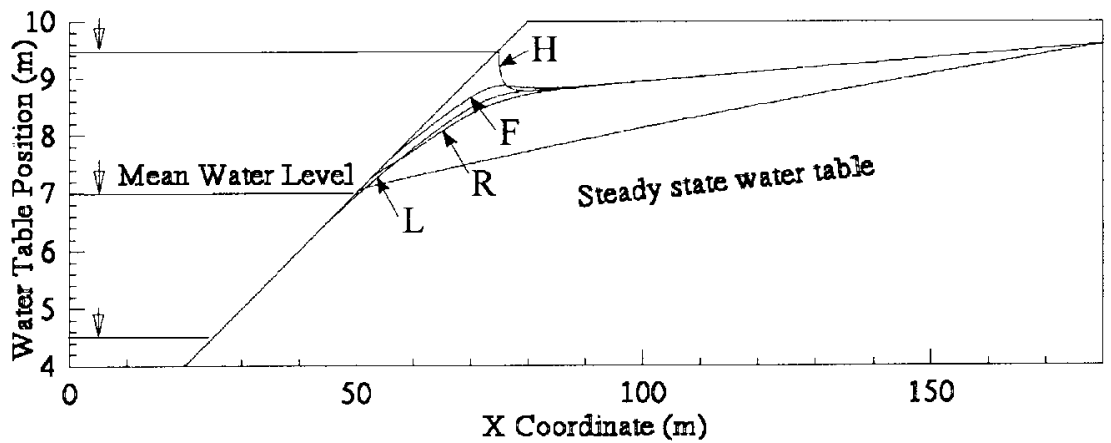

Figure 14. Water tables predicted at different stages of tidal level (case VI). H-high tide; F-falling tide; L=low tide; R-rising tide

a large tidal influence. The volume of this discharge during one tidal cycle is $0.33 \mathrm{~m}^{3}$ compared with the steady-state case of $0.70 \mathrm{~m}^{3}$.

\section{Influence of beach slope (case VII)}

To investigate the effect of beach slope on this process, a vertical face aquifer is also considered. The aquifer is $130 \mathrm{~m}$ long, which in the case of the sloping face aquifer is the horizontal distance between the land boundary and the intersection of sea with shoreline. The other parameters are the same as for the basic problem, except that the tidal amplitude is $2.5 \mathrm{~m}$. Although a vertical face is obviously the extreme case, it has been included because the vast majority of previous studies of groundwater discharge to the sea have assumed a vertical outflow face.

A finite-element mesh with 5589 nodes and 5354 elements is applied in the simulation. The element dimensions vary from $0.1 \mathrm{~m}$ in the unsaturated zone to $1 \mathrm{~m}$ in saturated parts of the aquifer. The time step, $\Delta t$, varies from 0.05 to $0.5 \mathrm{~h}$ in simulations.

Figure 15 shows the water table at different stages of a tidal cycle for this case. A comparison of Figure 15 and Figure 11 clearly indicates the importance of the beach slope on the processes. Obviously the tidal effect on the groundwater dynamics is much more significant for a mildly sloping beach than for a steeper one.

For the vertical face aquifer, the volume of groundwater discharged to the sea is $0.28 \mathrm{~m}^{3}$ over a full tidal cycle and in the steady-state case is $0.33 \mathrm{~m}^{3}$. The difference between the exit point and seawater level for 


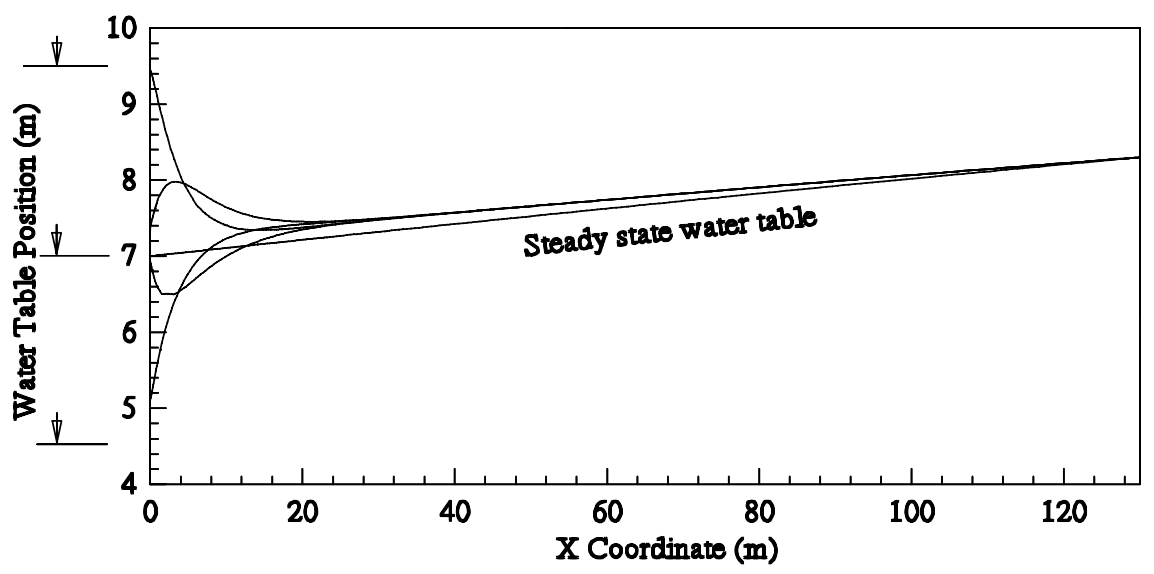

Figure 15. Water tables predicted at different stages of a tidal cycle (case VII)

the low-tide condition is $0.6 \mathrm{~m}$, which is much less than $1.66 \mathrm{~m}$ in the case of a sloping beach (Figure 13). Also the variation of discharge to sea during a period of oscillation is not as irregular as that in the case of a sloping beach (Figure 12).

\section{CONCLUSIONS}

A two-dimensional variably saturated numerical model was applied to simulate a hypothetical coastal aquifer. The effects of tidal fluctuation on the water flow in the aquifer were investigated assuming uniform fluid density. Simulations showed that the tidal fluctuation has substantial consequences for the local velocity field in the vicinity of the exit face. It was observed that there is an overheight in the water table resulting from tidal fluctuation and a long seepage face may be produced in cases with a large tidal amplitude. Also the velocity field is markedly different from that which occurs when the tide is ignored. In particular there are substantial vertical components of velocity when tidal effects are included, whereas without them the velocities are predominantly horizontal.

The effect of beach slope is very significant and simplifying the problem by considering a vertical beach face causes serious errors in results for the water-table position and groundwater flux for the case of a constant water level at the landward boundary. As shown for cases V and VII, the difference between the exit point and seawater level for the low-tide condition is $0.6 \mathrm{~m}$ for the vertical face aquifer (case VII), which is much less than $1.66 \mathrm{~m}$ in the case of a sloping beach (case V). For the vertical face aquifer, the volume of groundwater discharged to the sea is $0.28 \mathrm{~m}^{3}$ over a full tidal cycle. However, in the case of sloping beach the tidal fluctuations have reversed the hydraulic gradient from land-sea to sea-land and the volume of water discharged to the landward source in this case is $0.11 \mathrm{~m}^{3}$.

Unsaturated zone effects are important for media with high effective capillary fringe when determining the quasi-steady-state water table resulting from tidal fluctuations of sea level. Based on this investigation the increase in the effective capillary fringe causes a decrease in the quasi-steady-state water table overheight resulting from tidal fluctuation.

In general this study shows that the tidal fluctuations have significant effects on the amount of water discharged from the aquifer to the sea for cases where the landward end of the aquifer extends to a constant water-level reservoir. For example, neglecting the effects of tidal fluctuation for the basic problem (case I) causes a 39\% error in the amount of water discharged to sea. Obviously, a proper assessment of the amount of groundwater discharge and of the velocity pattern is essential to predict contaminant transport in groundwater and to manage the water quality at the coastline. 
It should be noted that although the investigations here have been for shallow aquifers, which are commonly an important source of freshwater in coastal areas and islands, the results and conclusions will be applicable for deeper aquifers, which are affected by large amplitude tides. For example the ratio of tidal amplitude to aquifer depth for the basic problem was 1:7, therefore the results will be relevant to an aquifer of $20 \mathrm{~m}$ depth that is influenced by a $3 \mathrm{~m}$ amplitude tide. A more complete analysis of tidal effects would include cases where tidal amplitude remains constant and depth of aquifer is varied.

\section{ACKNOWLEDGEMENTS}

The postgraduate scholarship of the Ministry of Culture and Higher Education of the Islamic Republic of Iran is appreciated. Financial assistance was provided partly by the Australian Research Council through a large grant for Coastal Groundwater Dynamics.

\section{REFERENCES}

Ataie-Ashtiani B, Volker RE, Lockington DA.1999a. Tidal effects on sea-water intrusion in unconfined aquifers. Journal of Hydrology 216(1-2): $17-31$.

Ataie-Ashtiani B, Volker RE, Lockington DA. 1999b. Numerical and experimental study of seepage in unconfined aquifers with a periodic boundary condition. Journal of Hydrology 222(1-4): 165-184.

Baird AJ, Horn DP. 1996. Monitoring and modelling groundwater behaviour in sandy beaches. Journal of Coastal Research 12(3): 630-640.

Celia MA, Bouloutas ET, Zarba RL. 1990. A general mass-conservative numerical solution for the unsaturated flow equation. Water Resources Research 26(7): 1483-1496.

Cooley RL. 1983. Some new procedures for numerical solution of variably saturated flow problems. Water Resources Research 19(5): $1271-1285$.

Dominick TF, Wilkins B, Roberts H. 1971. Mathematical model for beach groundwater fluctuations. Water Resources Research 7: $1626-1635$.

El-Kadi AI, Ling G. 1993. The Courant and Peclet number criteria for the numerical solution of the Richards equation. Water Resources Research 29(10): 3485-3494.

Fang CS, Wang SN, Harrison W. 1972. Groundwater flow in a sandy tidal beach: two dimensional finite element analysis. Water Resources Research 8: $121-128$.

Grant US. 1948. Influence of the water table on beach aggradation and degradation. Journal of Marine Research 7: 655-660.

Jacob CE. 1950. Flow of groundwater. In Engineering Hydraulics, Rouse H (ed.). Wiley: New York; 321-386.

Knight JH. 1981. Steady periodic flow through a rectangular dam. Water Resources Research 17(4): 1222-1224.

Li L, Barry DA, Pattiaratchi CB. 1997. Numerical modelling of tidal-induced beach water table fluctuations. Coastal Engineering 30: $105-123$.

Neuman SP. 1973. Saturated-unsaturated seepage by finite elements. Journal of the Hydraulics Division, American Society of Civil Engineers 99(Hy12): 2233-2250.

Nielsen P. 1990. Tidal dynamics of the water table in beaches. Water Resources Research 26(9): 2127-2134.

Nielsen P, Aseervatham R, Fenton JD, Perrochet P. 1997. Groundwater waves in aquifers of intermediate depths. Advances in Water Resources 20(1): 37-43.

Parlange JY, Stagnitti F, Starr JL, Braddock RD. 1984. Free surface flow in porous media and periodic solution of the shallow flow approximation. Journal of Hydrology 70: 251-263.

Philip JR. 1973. Periodic non-linear diffusion: an integral relation and its physical consequences. Australien Journal of Physics 26: 513-519.

Smiles DE, Stokes AN. 1976. Periodic solutions of a nonlinear diffusion equation used in groundwater flow theory: examination using a Hele-Shaw model. Journal of Hydraulics 31: 27-35.

Turner IL, Coates BP, Acworth RI. 1996. The effects of tides and waves on water-table elevations in coastal zones. Hydrogeology Journal 4(2): $51-69$.

Van Genuchten MTh., 1980. A closed-form equation for predicting the hydraulic conductivity of unsaturated soils. Soil Society of America Journal 44: 892-898.

Voss CI. 1984. SUTRA: a Finite Element Simulation Model for Saturated-Unsaturated, Fluid-density Dependent Ground-water Flow with Energy Transport or Chemically Reactive Single Species Solute Transport. US Geological Survey, National Centre: Reston, VA.

Wise WR, Clement TP, Molz FJ. 1994. Variably saturated modeling of transient drainage: sensitivity to soil properties. Journal of Hydrology 161: $91-108$. 